\title{
Coronary care programme improved food habits but not physical activity or smoking status after acute myocardial infarction
}

\author{
Carlsson $R$, Lindberg $G$, Westin L, et al. Influence of coronary nursing management follow up on lifestyle after acute myocardial \\ infarction. Heart 1997 Mar;77:256-9.
}

\section{Objective}

To determine whether a secondary prevention programme run by a nurse rehabilitator improves the lifestyle (food habits, smoking status, and physical activity) of adults during the year after an acute myocardial infarction.

\section{Design}

Randomised controlled trial with 1 year follow up.

\section{Setting}

A university hospital in Sweden.

\section{Patients}

168 adults (mean age 62 y, $75 \%$ men) who were $\geqslant 50$ years old and admitted to hospital with a confirmed acute myocardial infarction (World Health Organisation definition). Exclusion criteria were unstable angina, predefined ST segment changes during exercise testing, or evidence of congestive heart failure.

\section{Intervention}

During the 3 weeks after hospital discharge, patients visited a nurse and cardiologist and received exercise training and information on risk factors and lifestyle changes. 4 weeks after discharge, patients were allocated to an intervention programme $(n=87)$ or usual care involving 2 general practitioner visits $(\mathrm{n}=81)$. The intervention programme consisted of visits with a nurse and cardiologist, a 3 month education programme (1.5 hours of smoking cessation training; 5.5 hours of dietary education for both spouses; 2 hours of physical activity education), and a 10-12 week exercise programme (40 minutes of interval training with jogging and cycling 2-3 times/wk). Follow up was $98 \%$.

\section{Main outcome measures}

Self reported physical activity ( 5 categories from sedentary to vigorous physical training), smoking habits (5 levels from never smoked to currently smoking), and food habits (5 levels). Data were dichotomised before analysis (sedentary $v$ physically active, not caring $v$ caring about food habits, and present smoking $v$ not smoking).

\section{Main result \\ More patients in the intervention group than in the usual care group reported that they cared about their food habits 1 year after their myocardial infarction $(89 \% v 62 \%, \mathrm{p}=0.008)$. The groups did not differ for stopping physical training (12\% for intervention group $v 17 \%$ for control group, $\mathrm{p}=0.43$ ), starting physical training $(78 \%$ for intervention group $v 67 \%$ for control group, $\mathrm{p}=0.5)$, or stopping smoking $(50 \%$ for the intervention group $v 29 \%$ for the control group, $\mathrm{p}=0.09$ ).}

\section{Conclusion}

A multicomponent coronary care programme run by nurses with input from cardiologists and physiotherapists designed for patients after an acute myocardial infarction improved food habits but had no effect on physical activity or smoking status.

Sources of funding: Swedish Heart Lung Foundation; Ernold Lundströms Foundation; Merck and Sharp and Dome Foundation.

For article reprint: $\mathrm{Dr} R$ Carlsson, Section of Cardiology, Department of Medicine, Central Hospital, S-651 85 Karlstad, Sweden. Fax +46054015349.

\section{Commentary}

The effectiveness of nurse monitored prevention programmes after myocardial infarction has been studied with inconsistent results. ${ }^{12}$ In their study, Carlsson $e t$ al included a control group, randomly allocated patients to groups, collected baseline and outcome measures, and studied a reasonably large sample for a relatively long follow up period of 1 year.

I have 2 concerns. During the first 3 months after randomisation, the intervention group participated in a 3 month education programme delivered by a nurse and 10-12 weeks of exercise training. Over the next 6 months, these patients had 3 additional visits with the nurse. 3 months later, the follow up data were collected; however, during this time no further intervention occurred. Without outcome measures for both groups at the end of the training programme, after the last visit with the nurse, and after a longer period of follow up, the results of this study are equivocal. It is possible that important gains were achieved during the education and exercise programmes, but relapse occurred after these programmes ended. It is possible that gains that occurred during these programmes were maintained during the period of visits with the nurse, but relapse occurred after the last visit. Even if effects had been seen immediately after these programmes ended, however, they would only have been transient and what is needed is programmes with lasting effects.

The second issue relates to the improvement in dietary habits. Given that these data were self reported without any attempt to confirm their accuracy, the findings may be suspect. The validity issue is especially important because the sub jects were not blinded to group assignment. It is possible that reported dietary attitudes of the intervention group were influenced by a desire to please assessors or to prove the participants' ability to maintain the regimen.

The jury is still out regarding the effectiveness of nurse monitored prevention programmes for patients with myocardial infarction. Future randomised trials are required building on the work of Carlsson et al.

\section{Michael Brand, RN, MS Information Specialist, San Antonio Cochrane Center San Antonio, Texas, USA}

1 Pozen MW, Stechmiller JA, Harris W, et al. A nurse rehabilitator's impact on patients with myocardial infarction. Med Care 1977; 15:830-7.

2 Burgess AW, Lernr DJ, D'gostino RB, et al. A randomised control trial of cardiac rehabilitation. Soc Sci Med 1987;24:359-70. 